\title{
Pebbling In Watkins Snark Graph
}

\author{
Ms.Sreedevi $\mathrm{S}^{1}$, Dr.M.S Anilkumar ${ }^{2}$, \\ Department of Mathematics, Mahatma Gandhi College, Thiruvananthapuram, Kerala ${ }^{1,2}$ \\ Email:sreedevi.sasikumar@gmail.com ${ }^{1}$,anilkumar250365@gmail.com²
}

\begin{abstract}
The pebbling theory is a study of a mathematical game that is played over a graph. In this game, a pebbling movement means removing two pebbles from a given vertex and adding one pebble to one of its neighbors and removing the other pebble from the game. The pebbling number of a graph $G$ is defined a smallest positive integer required to add a pebble at any target vertex of the graph. It is denoted as $\pi(G)$. Every vertex of the graph is pebbled irrespective of the initial pattern of pebbles. Cubic graph is also called a 3- regular graph which is used in a real time scenario. In this paper, we have determined the pebbling number of Watkins Snark by constructing a Watkins Flower Snark of vertices, edges, cycles and disjoint sets which are present in the Watkins Snark. It is a connected graph in which bridgeless cubic index is equal to 4 with 75 edges and 50 vertices.
\end{abstract}

Keywords - Graphs; pebbling number; Watkins Snark graph

\section{INTRODUCTION}

Throughout this paper, let $G=(V, E)$ denotes a simple connected graph. $n=|V|$ and $m=|E|$ are the number of vertices and edges respectively in $\mathrm{G}$ and the diameter of $\mathrm{G}$ is denoted as $d$. The weights assigned to the vertices of a graph are a non-negative integer which might represent a discrete resource.

Lagarias and Saks first suggested the idea of pebbling in graphs to solve specific problems in number theory. In 1989 Chung [1] defined the pebbling number for any graph $G$.

\subsection{Pebbling move}

A pebbling move on a graph means taking two pebbles off from the particular vertex and placing one of the pebbles at any neighboring vertex and eliminating another pebble from the game.

\subsection{Graph pebbling}

Graph pebbling is a branch of graph theory, which is considered as a game to play on a graph with a given distribution of pebbles over the vertices of the graph.

For example, consider two players playing the mentioned game. Player A distributes the pebbles over the vertices of the graph and asks Player B to reach the target vertex by making a sequence of pebbling moves if player B reaches the target, either he wins or player A wins. In such a game, the intention of the study is to find the minimum number of pebbles required to distribute over the vertices so that the player wins

\subsection{Solvability}

Consider Peterson graph with pebbles on the vertices. From the (Figure-1), one can move 2 pebbles among 3 pebbles from the vertex and add 1 pebble to the neighboring vertex. Such a distribution of pebbles on vertex over a graph is solvable. However from (Figure2 ), the movement of the pebble is not possible at any vertices, but every vertex contains either 0 or one pebble. Also the pebbling movement will be possible only if 2 pebbles are available at any one of the vertex.

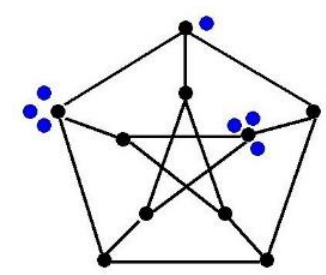

Figure-1: Solvable

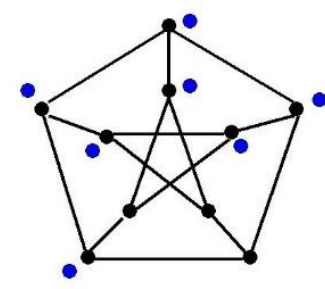

Figure-2: Unsolvable

\subsection{Pebbling number}

In a graph $G=(V, E)$, the smallest possible natural number $n$ required to obtain a new configuration in which the target or root vertex has one or more pebbles after a series of pebbling which moves from the initial configuration. This pebbling movement is denoted by $\pi(G)$. It denotes the fewest number of pebbles independent from the initial configuration. Pebbling numbers of a path, cycle and wheel graphs over $n$ vertices are given below

$\pi\left(K_{n}\right)=n$ 


\section{Available online at www.ijrat.org}

$\pi\left(P_{n}\right)=2^{n-1}$

$\pi\left(W_{n}\right)=n$

\subsection{Watkins Snark graph}

Watkins Snark Graph is a Snark in graph theory, discovered by John J. Watkins in 1989. He used 50 vertices and 75 edges to describe the snark. It denotes that $J_{50}$ is a graph with vertex set $V\left(J_{50}\right)=V_{1} \cup V_{2}$ where $V_{1}=\left\{a_{i}: i=1,2,3,4, \ldots \ldots, 25\right\}, V_{2}=$ $\left\{b_{i}: i=1,2,3,4, \ldots, 25\right\}=V_{5}^{1} \cup V_{5}^{2} \cup V_{5}^{3} \cup V_{5}^{4} \cup V_{5}^{5}$

$V_{5}^{1}=\left\{b_{1}, b_{19}, b_{2}, b_{20}, b_{3}\right\}, V_{5}^{2}=\left\{b_{11}, b_{4}, b_{12}, b_{5}, b_{13}\right\}$

$V_{5}^{3}=\left\{b_{14}, b_{22}, b_{15}, b_{23}, b_{21}\right\}, V_{5}^{4}=\left\{b_{24}, b_{7}, b_{25}, b_{8}, b_{6}\right\}$

$V_{5}^{5}=\left\{b_{9}, b_{17}, b_{10}, b_{18}, b_{16}\right\}$

And edge set $E=E_{1} \cup E_{2}$

where $E_{1}=\left\{e_{i}^{1}=a_{i} a_{i+1}: i=1,2,3, \ldots, 24\right\}$

$$
E_{2}=\left\{e_{i}^{1}=a_{i} b_{i}: i=1,2,3, \ldots, 25\right\}
$$

$$
\cup E_{5}^{1} \cup E_{5}^{2} \cup E_{5}^{3} \cup E_{5}^{4} \cup E_{5}^{5}
$$

where $E_{5}^{1}=\left\{b_{1} b_{19}, b_{19} b_{2}, b_{2} b_{20}, b_{20} b_{3}, b_{3} b_{1}\right\}$,

$$
\begin{aligned}
E_{5}^{2} & =\left\{b_{11} b_{4}, b_{4} b_{12}, b_{12} b_{5}, b_{5} b_{13}, b_{13} b_{11}\right\}, \\
E_{5}^{3} & =\left\{b_{21} b_{14}, b_{14} b_{22}, b_{22} b_{15}, b_{15} b_{23}, b_{23} b_{21}\right\} \\
E_{5} & =\left\{b_{6} b_{24}, b_{24} b_{7}, b_{7} b_{25}, b_{25} b_{8}, b_{8} b_{6}\right\},
\end{aligned}
$$

where $V_{5}^{1}, V_{5}^{2}, V_{5}^{3}, V_{5}^{4}, V_{5}^{5}$ and $E_{5}^{1}, E_{5}^{2}, E_{5}^{3}, E_{5}^{4}, E_{5}^{5}$ are disjoint sets of vertices and edges of cycles $C_{5}^{1}, C_{5}^{2}$, $C_{5}^{3}, C_{5}^{4}, C_{5}^{5}$ respectively. In Fiure-3, Watkins Snark set of vertices $V_{1}$ forms a cycle of $C_{25}$ numbers.

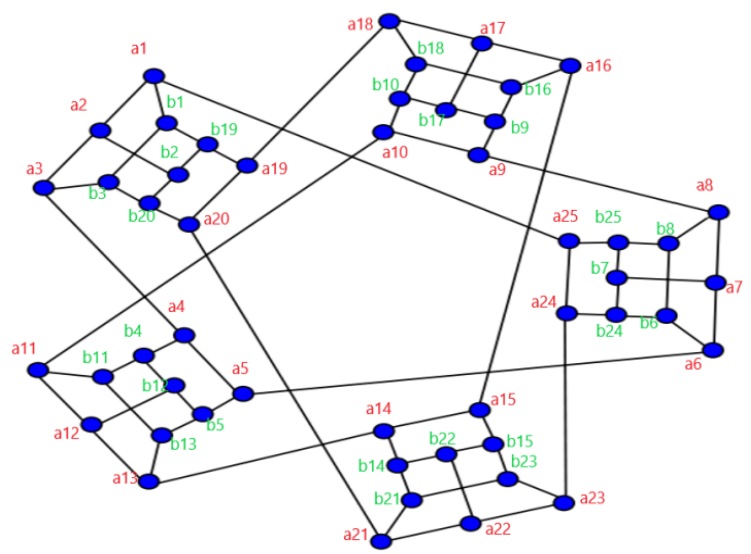

Figure-3: Watkins Vertex set

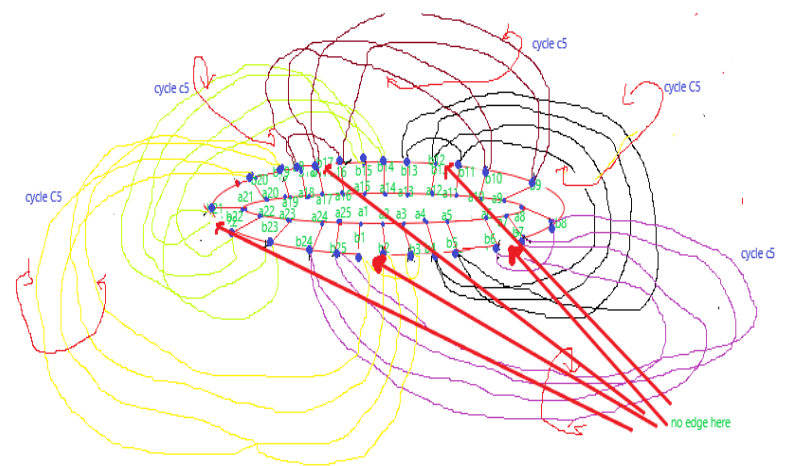

Figure-4: Flower Watkins Snark (Edge set)

Pebbling on graphs is a two-player game on a connected graph, first introduced by Lagarias and Saks. Later Chung [1] gave the new introduction for the two-player game. In [2], Pachter et al. proved that every graph of diameter two on $\mathrm{N}$ vertices has a pebbling number either $\mathrm{N}$ or $\mathrm{N}+1$.. In [3], A. Lourdusamy et al. introduced the generalized pebbling number of a graph. David Moews [4] proved that the pebbling number of the product of two graphs $f(G \times H) \leq f(G) f(H)$.Ye et al. [5] determined the pebbling numbers of squares of even cycles. Whereas Asplund et al. [6] showed $\pi(G \times H) \leq$ $(\pi(G)+|G|) \pi(H)$ and provided similar results for other graph products and graph operations. Melody Chan et al. [7] upper bounded by considering the configuration of pebbles distributed on the vertices of a connected graph of order $\mathrm{n}$. If $\mathrm{G}$ is a connected graph with $\mathrm{n}$ vertices and $\delta(G)=k$, then optimal pebbling $\pi^{*}(G) \leq \frac{4 n}{k+1}$. For Watkins Snark $J_{50}, k=3$ and $n=50$,

$\pi^{*}\left(J_{50},\right) \leq \frac{4 \times 50}{3+1}=50$ thus $\pi^{*}\left(J_{50},\right)=50$.

$\max \left\{n, 2^{d}\right\} \leq \pi(G) \leq(n-1)\left(2^{d}-1\right)+1$

(2), Melody Chan et al. [7] proposed the new upper bound as,

$\pi(G) \leq(n-d)\left(2^{d}-1\right)+1$

In (3),Melody Chan et al. [7] improved the upper bound of the theorem (2)

$$
f(G) \leq\left(n+\left\lfloor\frac{n-1}{d}\right\rfloor-1\right)\left(2^{d-1}\right)-n+2
$$




\section{Available online at www.ijrat.org}

In (3), Melody Chan et al. [7]proved that $\mathrm{G}$ has an efficient dominating set of size $\gamma$.

\section{LOWER AND UPPER BOUNDS OF PEBBLING NUMBER OF WATKINS SNARK}

Watkins graph has $d=\operatorname{diam}\left(J_{50}\right)=7$ and size $\mathrm{n}=$ 50. Thus

$$
\begin{aligned}
& \max \left\{50,2^{7}\right\} \leq \pi\left(J_{50}\right) \leq(50-1)\left(2^{7}-1\right)+1 \\
& \max \{50,128\} \leq \pi\left(J_{50}\right) \leq(49)(127)+1
\end{aligned}
$$$$
128 \leq \pi\left(J_{50}\right) \leq 6224
$$

From Eq. 4, improved bounds become

$$
\begin{aligned}
& \pi(G) \leq(50-7)\left(2^{7}-1\right)+1=5462 \\
& 128 \leq \pi\left(J_{50}\right) \leq 5462
\end{aligned}
$$

From $E q .5$, new bounds become

$$
\begin{aligned}
& \pi\left(J_{50}\right) \leq\left(50+\left\lfloor\frac{50-1}{7}\right\rfloor-1\right)\left(2^{7-1}\right)-50+2 \\
& \pi\left(J_{50}\right) \leq 3536
\end{aligned}
$$$$
128 \leq \pi\left(J_{50}\right) \leq 3536
$$

Theorem.2.1. In (5), Bukh Boris [8] proved that pebbling number of a graph with $n$ vertices and diameter d satisfies

$\pi(n, d) \leq\left(2^{\left\lceil\frac{d}{2}\right\rceil}-1\right) n+O(\sqrt{n})$

Theorem.2.2. In (7), Postle [9]showed that if diameter d of a connected graph with $\mathrm{n}$ vertices is odd, then

$\pi(G) \leq f\left(\left\lceil\frac{d}{2}\right\rceil\right) n+O(1)$, where $\mathrm{f}(\mathrm{k})=\frac{\left(2^{k}-1\right)}{k}(E q .11)$

For Watkins Snark $J_{50}$,

$$
\begin{aligned}
& \pi\left(J_{50}\right) \leq f\left(\left\lceil\frac{7}{2}\right\rceil\right) 50+O(1)=50 f(4)+O(1), \\
& f(4)=\frac{\left(2^{4}-1\right)}{4}=\frac{15}{4} \\
& \pi\left(J_{50}\right) \leq 50\left(\frac{15}{4}\right)+O(1)=187.5+O(1) \\
& 128 \leq \pi\left(J_{50}\right) \leq 187.5+C
\end{aligned}
$$

where $\mathrm{C}$ is a constant.

\section{PEBBLING NUMBER OF WATKINS SNARK}

\subsection{Pebbling number of Watkins Snark $J_{50}$.}

A Watkins Snark $J_{50}$ is a graph whose vertex set is partitioned into two disjoints subsets $V_{1}$ and $V_{2}$ where $V_{1}=\left\{a_{1}, a_{2}, a_{3}, \ldots \ldots a_{25}\right\}$ and

$V_{2}=\left\{b_{1,}, b_{2}, b_{3}, \ldots \ldots b_{25}\right\}$

Set $V_{2}$ can be again portioned as

$$
\begin{aligned}
& V_{2}^{1} \cup V_{2}^{2} \cup V_{2}^{3} \cup V_{2}^{4} \cup V_{2}^{5} \text { where } \\
& V_{2}^{1}=\left\{b_{1}, b_{25}, b_{24}, b_{23}, b_{22}\right\} \\
& V_{2}^{2}=\left\{b_{2}, b_{3}, b_{4}, b_{5}, b_{6}\right\} \\
& V_{2}^{3}=\left\{b_{7}, b_{8}, b_{9}, b_{10}, b_{11}\right\}, \\
& V_{2}^{4}=\left\{b_{12}, b_{13}, b_{14}, b_{15}, b_{16}\right\} \\
& V_{2}^{5}=\left\{b_{17}, b_{18}, b_{19}, b_{20}, b_{21}\right\} .
\end{aligned}
$$

Every vertex $a_{i} \in V_{1}$ is adjacent to vertex $b_{i} \in V_{2}$ for all $1 \leq i \leq 25$. The vertex sets of the cycle $C_{5}$ are given below:

$V_{C_{5}}^{1}=\left\{b_{1}, b_{19}, b_{2}, b_{20}, b_{3}\right\}, V_{C_{5}}^{2}=\left\{b_{11}, b_{4}, b_{12}, b_{5}, b_{13}\right\}$ , $V_{C_{5}}^{3}=\left\{b_{14}, b_{22}, b_{15}, b_{23}, b_{21}\right\}$,

$V_{C_{5}}^{4}=\left\{b_{24}, b_{7}, b_{25}, b_{8}, b_{6}\right\}, V_{C_{5}}^{5}=\left\{b_{9}, b_{17}, b_{10}, b_{18}, b_{16}\right\}$ are subsets of $V_{2}$.

Denoting the number of pebbles distributed over each vertex of $V_{j}$ for all $j=1,2$ by $p_{j}$, equation $\mathrm{A} .12$ is given as,

$$
p_{2}=p_{2}^{1}+p_{2}^{2}+p_{2}^{3}+p_{2}^{4}+p_{2}^{5}
$$

where $p_{2}^{j}$ denotes the number of pebbles distributed over each vertex of $V_{2}^{j}$ for all $j=1,2,3, \ldots, 5$.

Let the number of pebbles initially placed on a particular vertex $x_{j}$ is denoted by $\mathrm{p}\left(x_{j}\right)$ for all $1 \leq j \leq 50$. Let the total number of pebbles on the set $V_{j}$ for all $j=1,2$ is denoted by $p(j)$. So

$p(2)=p^{1}(2)+p^{2}(2)+p^{3}(2)+p^{4}(2)+p^{5}(2)$ 


\section{International Journal of Research in Advent Technology, Vol.7, No.2, March 2019 \\ E-ISSN: 2321-9637 \\ Available online at www.ijrat.org}

where $p^{j}(2)$ denotes the total number of pebbles on the set $V_{2}^{j}$ for all $j=1,2,3, \ldots, 5$. There are two possible cases of sets where the target vertex may belong $V_{1}$ and $V_{2}$.

\section{Case 1:}

In this case, the target vertex $a_{1} \in V_{1}$. If $p\left(a_{2}\right)=2$ or $p\left(a_{25}\right)=2$ determines the pebbling movement either from $a_{2}$ or $a_{25}$, then only 2 pebbles are necessary to reach the target that is trivial. So assume that

$\mathrm{p}\left(a_{2}\right)<2, p\left(a_{25}\right)<2$

Case 1.1: $p_{1}>1$ :

In this case, assume that the vertex of the set $V_{1}$ would distribute at least two pebbles in the vertex. Then for some $i$,such that $p\left(a_{i}\right) \geq 2$ for $3 \leq i \leq 24$ which would start the first step in pebbling. Then the pebbling sequence would reach the target vertex which is given as $\left\{a_{i}, a_{i-1}, \ldots, a_{2}, a_{1}\right\}$. On placing two pebbles on each of $25-3=22$ vertices of $V_{1}$ and in particular at least one pebble on $a_{2}$ and $a_{25}$, the sum of the total number of pebbles required to reach the target vertex $a_{1}$ is

$$
p^{1} \geq 2 \times 22+2=46
$$

The target can also be reached by placing $2^{d}$ pebbles on a single vertex, where $\mathrm{d}$ is the diameter to the target vertex. Since set $V_{1}$ forms an odd cycle $C_{25}$, the required number of pebbles $V_{1}$ to pebble the target vertex $a_{1}$ is given by

$$
\begin{gathered}
\pi\left(C_{25}\right)=2\left\lfloor\frac{2^{d+1}}{3}\right\rfloor+1=2\left\lfloor\frac{2^{12+1}}{3}\right\rfloor+1 \\
=5461
\end{gathered}
$$

\section{Case 1.2: $p_{1} \leq 1$ :}

In this case, the set $V_{1}$ has insufficient number of pebbles to start a pebbling step. In order to reach the target $a_{1}$, we need to extract the pebbles from the set $V_{2}$. The following sub cases are possible ways to extract the pebbles from $V_{2}$. In these sub cases, assume that $p\left(b_{1}\right)=0$ which avoids the trivial pebbling numbers.

Case 1.2.1: $p_{2} \geq 2$ :

In this case, suppose $p_{2} \geq 2 \Rightarrow p_{2}^{j} \geq 2$ for all $\mathrm{j}$ $=1,2,3,4,5$, then each vertex $a_{i}$ is adjacent to vertex $b_{i}$ for all $i \in\{1,2, \ldots 25\}$. Consider $p\left(b_{1}\right)=0$, since $p_{2} \geq 2$, one pebble can be moved to $V_{1}$ so that $p_{1} \geq 2$. After these pebbling moves, the target vertex is reached using the case 1.1 When $p_{1}=0$, there will be no vertex in $V_{1}$ in order to make the pebbling movement. Hence to start the pebbling movement $i$ should be in $2 \leq i \leq$ 25 such that $p\left(b_{i}\right) \geq 4$ allows two pebbles moved to vertex $a_{i} \in V_{1}$ and the sequence of pebbling moves would be $\left\{b_{i}, a_{i}, a_{i-1}, a_{i-2}, \ldots a_{2}, a_{1}\right\}$ or

$\left\{b_{i}, a_{i}, a_{i+1}, a_{i+2}, \ldots a_{25}, a_{1}\right\}$ for $2 \leq i \leq 25$. Thus there are at most one pebble on 24 vertices of $V_{1}$ and minimum two pebbles on 24 vertices of $V_{2}$, so the total number of pebbles required to reach the target vertex is

$p^{1}+p^{2} \geq 24+2=72$

Case 1.2.2: $p_{2}^{1} \leq 1$

Since $p_{2}^{1} \leq 1$, set $V_{2}^{1}$ has insufficient number of pebbles to move one pebble to some vertex of $V_{1}$.

To make a pebbling move a set of $V_{2}^{k}$ pebbles must be extracted where $k \boldsymbol{\epsilon}\{\mathbf{2}, \mathbf{3}, \mathbf{4}, \mathbf{5}\}$ which is discussed in following sub cases.

\section{Case 1.2.2.1: $p_{2}^{k} \geq 2$ for some $k \epsilon\{2,3,4,5\}$ :}

As every $b_{i} \in V_{2}$ belongs to cycle $C_{5}$, for example, $b_{1} \in C_{5}=\left\{b_{1}, b_{19}, b_{2}, b_{20}, b_{3}\right\}$.Through this cycle the pebbles can be extracted from $V_{2}^{l}$ for some $l \in\{2,3,4,5\}$ by making the pebbling sequence of length at most 5 , i.e. a path of length 5

Assume $p\left(x_{i}\right) \geq 2$ for each $x_{i} \in\left\{b_{1}, b_{19}, b_{2}, b_{20}, b_{3}\right\}$

We know pebbling number of a path $P_{5}$ is given by

$$
\pi\left(P_{5}\right)=2^{5-1}=2^{4}=16
$$

Which denotes the required number of pebbles to pebble the target vertex $x_{1}$ is greater than the minimum number of pebbles. Therefore it is found that there are at most one pebble in 25 vertices of $V_{1}$ and if 16 pebbles are placed at the path, 1 pebble is distributed at each vertex of $V_{1}$ except the cycle. Hence the minimum number of pebbles required are $p_{1}+20+16=25+36=61$. If

$$
p\left(x_{i}\right) \leq 1 \text { for each } x_{i} \in\left\{b_{1}, b_{19}, b_{2}, b_{20}, b_{3}\right\} .
$$

then $p\left(x_{i}\right)=1$. In order to move a pebble to $a_{1}$ the pebbles have to be extracted from other cycles, $C_{5} \neq$ $\left\{b_{1}, b_{19}, b_{2}, b_{20}, b_{3}\right\}$. From the extracted pebbles from other cycles, a pebbling sequence of 8 vertices is needed i.e., a path of length 8 and the known pebbling number of a path length $\mathrm{n}$ is $\pi\left(P_{n}\right)=2^{8-1}=2^{7}=128$. 


\section{International Journal of Research in Advent Technology, Vol.7, No.2, March 2019 \\ E-ISSN: 2321-9637 \\ Available online at www.ijrat.org}

In this case, 1 pebble at 25 vertices of $V_{1}, 1$ pebble at each vertex of the cycle and 128 pebbles at the path of 8 vertices and 1 pebble at each of remaining 13 vertices of $V_{2}$. Hence, in this case, the minimum number of pebbles required is

$$
\begin{gathered}
p_{1}+1 \times(13)+128=25+1 \times 13+128 \\
=166
\end{gathered}
$$

Let $p\left(x_{i}\right)=0, \quad$ in this case for each $x_{i} \in\left\{b_{1}, b_{19}, b_{2}, b_{20}, b_{3}\right\}$, there will be no vertex in order to make a pebbling movement. The pebbling sequence must be through the vertices of $V_{1}$ which have 8 vertices. For example to reach target vertex $a_{1}$ and all the elements at the cycle having 0 pebble a pebbling sequence $\quad\left\{b_{19}, a_{19}, a_{18}, a_{19}, a_{20}, a_{21}, a_{22}, a_{23}, a_{24}\right\}$ is made.

\section{Case 2:}

In the second possibility of the target, vertex would be a vertex set which is identified as $V_{2}$. So let $b_{1} \in V_{2}$ be the target vertex. Each vertex $b_{i} \in V_{2}$ is adjacent to 5 and vertices except $b_{1}, b_{2}, b_{6}, b_{7}, b_{11}, b_{12}, b_{16}, b_{17}, b_{21}, b_{22}$ are adjacent to 4 vertices. Our target vertex $b_{1}$ is adjacent to 4 vertices. So assumption can be made to avoid the trivial pebbling step as follows,

$p\left(a_{1}\right)=1, p\left(b_{3}\right)=1, p\left(b_{19}\right)=1, p\left(b_{25}\right)=1(E q .21)$

Case 2.1: $p_{2} \geq 2$

In this case, pebbles are to be transmitted from any arbitrary vertex $b_{i} \in V_{2}$ to reach the target vertex $b_{1} \in V_{2}$. Each vertex $b_{i} \in C_{5}$ is adjacent to vertex $a_{i}$ for $i=$ $1,2,3, \ldots \ldots, 25$. In order to start the pebbling move, it is necessary to extract the pebbles from $V_{1}$. So the minimum number of pebbles required can be denoted as the equation Eq. 22.

$p_{1}+p_{2}=2 \times 24+1 \times 22=70$

\section{Case 2.2: $p_{2} \leq 1$}

When $\boldsymbol{p}_{2}=\mathbf{1}$, pebbles will be extracted from $V_{1}$. Assume $\boldsymbol{p}_{\mathbf{1}}=\mathbf{2}$, then one pebble can be moved to the vertices of $V_{2}$ and the target vertex is pebbled. So the required minimum number of pebbles is given as,

$$
p_{1}+p_{2}=22+2 \times 24+4=74
$$

Let $\mathrm{p}_{2}=0$. This allows two pebbles moved to $b_{1}$. In this case, pebbles can be moved through a cycle $C_{5}$ from $V_{1}$. Pebbling number of $C_{5}$ is
$\pi\left(C_{5}\right)=2\left\lfloor\frac{2^{2+1}}{3}\right\rfloor+1=5$

So the required minimum number of pebbles is

$p_{2}+p_{1}=2 \times 24+5=52$

\section{Case 2.2.1: $p_{2} \leq 1$}

Here, extraction of pebbles takes place from $V_{1}$. In this case, $V_{2}$ have an insufficient number of pebbles. So we need to extract pebbles from the set $V_{1}$. Assume $p_{2}^{1} \leq 1$. In this case, $V_{2}^{1}$ has the insufficient number of pebbles to make pebbling move to target vertex $b_{1}$. So we can extract from $\boldsymbol{V}_{\mathbf{1}}$ through cycle $C_{5}$. Hence we need to make a pebbling sequence of 8 vertices, i.e. a path of length 8 and minimum number of pebbles required in a path graph length $\mathrm{n}$ is

$\pi\left(P_{n}\right)=2^{8-1}=2^{7}=128$

In this case, 1 pebble is at 25 vertices of $V_{2}, 1$ pebble at each vertex of the cycle and 128 pebbles at the path of 8 vertices and 1 pebble at each of remaining 13 vertices of $V_{2}$. Hence the minimum number of pebbles required as the target pebble is

$p_{1}+1 \times(13)+128=25+1 \times 13+128=166$

On comparing the case (2.1) and (2.2), the set of possible minimum pebbling numbers is obtained as $\{46,72,61,166\}$ in case (2.1). In case (2.2) it is obtained as $\{70,52,166\}$.From the equation (Eq. A.29), as all the possibilities of pebbling the target vertex in the sets $V_{1}, V_{2}$ are discussed, the equation becomes

$128 \leq \pi\left(J_{50}\right) \leq 187.5+C$

On considering the least possibility of the pebbling number from the two cases, it is concluded that the least possibility of $\{46,72,61,166\}$ and $\{70,52,166\}$ is 166 . Thus

$\pi\left(J_{50}\right)=166$

\section{CONCLUSIONS}

In this study, the pebbling number of Watkins Snark is calculated by constructing a Watkins Flower Snark by edges, vertices, cycles and disjoint sets present in the graph. By applying the methodology, the pebbling number of graphs is determined as Double-star snark, Szekeres snark, Loupekine snark, Fullerene graphs. The applications of pebbling are in positional games such as "Cops-and-Robbers" and "Chip-Firing." The pebbling 


\section{Available online at www.ijrat.org}

methodology of these games can be used in structural graph theory and theoretical computer science. Theory of pebbling can be applied in a toll or as a loss of information, fuel or electrical charge. The concept of graph pebbling can be generalized as q-pebbling and as the rate of loss. We can choose any constant rate $\alpha$ of loss instead of integer values in the initial distribution of pebbles. In a pebbling step one removes weight $w$ from one vertex and places weight $\alpha w$ at an adjacent vertex, for any constant $0<\alpha<1$. The objective of the study is still to place weight 1 at any prescribed root $r$ so that there is enough money, fuel, information, or energy at that location in the network. This generalized $\alpha$-pebbling is useful to make a chip-firing model. For any graph G, an auxiliary graph $\mathrm{H}$ can be obtained, so that chip-firing results on $\mathrm{H}$ can be brought on pebbling number of $\mathrm{G}$.

\section{REFERENCES}

[1] F.R.K. Chung, Pebbling in Hypercubes SIAM Journal on Discrete Mathematics, (1989), 467-472.

[2] L. Pachter, H. S. Snevily, and B. Voxman, On pebbling graphs, 1995.

[3] Lourdusamy and C. Muthu Lakshmi, Generalized Pebbling Number. International Mathematical Forum, 5 (2010) 1331 - 1337.

[4] David Moews, Pebbling Graphs. Journal of Combinatorial Theory, Series B 55, 24252 (1992).

[5] Y. Ye, P. Zhang, and Y. Zhang, The pebbling number of squares of even cycles. Discrete Mathematics, 312.21 (2012), 3203-3211.

[6] J. Asplund, Hurlbert, and F. Kenter, Pebbling on graph products and other binary graph constructions. arXiv preprint arXiv:1801.07808 (2018).

[7] Melody Chan, P. Anant, Godbole Note Improved pebbling bounds. Discrete Mathematics, 308 (2008), 2301-2306.

[8] Bukh, Boris, Maximum pebbling number of graphs of diameter three. Journal of Graph Theory, 52.4 (2006), 353-357.

[9] L. Postle, Pebbling graphs of fixed diameter. Journal of Graph Theory, 75.4 (2014), 302-310. 